Honam Mathematical J. 33 (2011), No. 3, pp. 393-405

http://dx.doi.org/10.5831/HMJ.2011.33.3.393

\title{
THE COMPLETE MOMENT CONVERGENCE FOR ARRAY OF ROWWISE ENOD RANDOM VARIABLES
}

\author{
DAE-HeE Ryu
}

\begin{abstract}
In this paper we obtain the complete moment convergence for an array of rowwise extended negative orthant dependent random variables. By using the result we can prove the complete moment convergence for some positively orthant dependent sequence satisfying the extended negative orthant dependence.
\end{abstract}

\section{Introduction}

Ebrahimi and Ghosh(1981) and Joag-Dev and Proschan(1983) introduced the concept of negative orthant dependent random variables:

A sequence $\left\{X_{i}, 1 \leq i \leq n\right\}$ of random variables is said to be negatively upper orthant dependent(NUOD) if for all real numbers $x_{1}, \cdots, x_{n}$,

$$
P\left(X_{1}>x_{1}, \cdots, X_{n}>x_{n}\right) \leq \prod_{i=1}^{n} P\left(X_{i}>x_{i}\right)
$$

and it is said to be negatively lower orthant dependent(NLOD) if for all real numbers $x_{1}, \cdots, x_{n}$,

$$
P\left(X_{1} \leq x_{1}, \cdots, X_{n} \leq x_{n}\right) \leq \prod_{i=1}^{n} P\left(X_{i} \leq x_{i}\right) .
$$

A sequence $\left\{X_{i}, 1 \leq i \leq n\right\}$ of random variables is said to be negatively orthant dependent(NOD) if it is both NUOD and NLOD.

Recently, Liu(2009) introduced the concept of extended negative dependence in the multivariate case. A sequence $\left\{X_{i}, 1 \leq i \leq n\right\}$ of

Received July 25, 2011. Accepted August 15, 2011.

2000 Mathematics Subject Classification. 60F15.

Key words and phrases. Extended negative orthant dependence, Complete convergence, Complete moment convergence, Positively orthant dependence. 
random variables is said to be extended negatively upper orthant depen$\operatorname{dent}(\mathrm{ENUOD})$ if for all real numbers $x_{1}, \cdots, x_{n}$, there exists a constant $M>0$ such that

$$
P\left(X_{1}>x_{1}, \cdots, X_{n}>x_{n}\right) \leq M \prod_{i=1}^{n} P\left(X_{i}>x_{i}\right)
$$

and it is said to be extended negatively lower orthant dependent(ENLOD) if for all real numbers $x_{1}, \cdots, x_{n}$, there exists a constant $M>0$ such that

$$
P\left(X_{1} \leq x_{1}, \cdots, X_{n} \leq x_{n}\right) \leq M \prod_{i=1}^{n} P\left(X_{i} \leq x_{i}\right) .
$$

A sequence $\left\{X_{i}, 1 \leq i \leq n\right\}$ of random variables is said to be extended negatively orthant dependent(ENOD) if it is both ENUOD and ENLOD.

It is clear that a sequence $\left\{X_{i}, 1 \leq i \leq n\right\}$ of random variables is called NOD if (1.3) and (1.4) hold when $M=1$, the sequence is called positively orthant dependent(POD) if the inequalities (1.3) and (1.4) hold both in the reverse direction when $M=1$. Obviously, an NOD sequence must be an ENOD sequence. On the other hand, for some POD sequences, it is possible to find a corresponding positive constant $M$ such that (1.3) and (1.4) hold.

Therefore, the ENOD structure is substantially more comprehensive than the NOD structure in which it can reflect not only a negative dependence structure also positive one to some extent. For instance, the ENOD sequence $\left\{X_{i}, i \geq 1\right\}$ in the following example can be taken as NOD or POD since there are no restrictions on the dependence between $X_{1}$ and $X_{2}$.

Example(Liu(2009)) If $\left\{X_{i}, i=1,2\right\}$ and $\left\{X_{i}, i \geq 3\right\}$ are independent of each other, where $X_{1}$ is possibly valued at $x_{11} \leq x_{12} \leq \cdots \leq x_{1 N}$ and $\left\{X_{i}, i \geq 3\right\}$ is a sequence of mutually independent random variables. Then the sequence $\left\{X_{i}, i \geq 1\right\}$ is ENOD. In fact, for any $x_{1}$ and $x_{2}$ such that

$$
P\left(X_{1} \leq x_{1}\right) P\left(X_{2} \leq x_{2}\right)=0 \text { or } P\left(X_{1}>x_{1}\right) P\left(X_{2}>x_{2}\right)=0
$$

both (1.3) and (1.4) hold trivially. Additionally, for any $x_{1}$ and $x_{2}$ such that

$$
P\left(X_{1} \leq x_{1}\right) P\left(X_{2} \leq x_{2}\right) \neq 0 \text { and } P\left(X_{1}>x_{1}\right) P\left(X_{2}>x_{2}\right) \neq 0,
$$

take

$$
M=1 / \min \left\{P\left(X_{1}=x_{1}\right), P\left(X_{1}=x_{1 N}\right)\right\},
$$


then both (1.3) and (1.4) still hold. Notice that there are no dependence restrictions between random variables $X_{1}$ and $X_{2}$.

A sequence of random variables $\left\{U_{n}, n \geq 1\right\}$ is said to converge completely to a constant $c$ if for any $\epsilon>0$,

$$
\sum_{n=1}^{\infty} P\left(\left|U_{n}-c\right|>\epsilon\right)<\infty .
$$

This notion was given by Hsu and Robbins(1947).

Let $\left\{Z_{n}, n \geq 1\right\}$ be a sequence of random variables and $a_{n}, b_{n}>0, q>$ 0 . If

$$
\sum_{n=1}^{\infty} a_{n} E\left\{b_{n}^{-1}\left|Z_{n}\right|-\epsilon\right\}_{+}^{q}<\infty \text { for all } \epsilon>0
$$

then (1.6) was called the complete moment convergence by Chow(1988).

Chow(1988) obtained the complete moment convergence for independent random variables. Wang and Zhao(2006) investigated for negatively associated random variables and $\mathrm{Zhu}(2007)$ studied for array of rowwise $\rho^{*}$-mixing random variables.

In this paper we study the complete moment convergence for partial sums of rowwise ENOD random variables.

\section{Some lemmas}

In this section we introduce some lemmas which will be used to prove the main result.

Lemma 2.1(Liu(2009)) Let $\left\{X_{i}, i \geq 1\right\}$ be a sequence of ENOD random variables.

(1) If $\left\{g_{i}(\cdot), i \geq 1\right\}$ is a sequence of monotone increasing(decreasing) functions, then $\left\{g_{i}\left(X_{i}\right), i \geq 1\right\}$ is still a sequence of ENOD random variables.

(2) If $X_{i}$ 's are nonnegative random variables, then there exists a constant $M>0$ such that

$$
E\left(\prod_{i=1}^{n} X_{i}\right) \leq M \prod_{i=1}^{n}\left(E X_{i}\right) .
$$

From (1) and (2) in Lemma 2.1 we obtain the following result. 
Lemma 2.2 Let $\left\{X_{i}, i \geq 1\right\}$ be a sequence of ENOD random variables. Then for any $h>0$ there exists a constant $M>0$ such that

$$
E\left[\exp \left(h \sum_{i=1}^{n} X_{i}\right)\right] \leq M \prod_{i=1}^{n} E\left[\exp \left(h X_{i}\right)\right]
$$

Proof. Note that $\left\{\exp \left(h X_{i}\right), i \geq 1\right\}$ is ENOD sequence by Lemma 2.1 (1). Hence by Lemma 2.1 (2) we have

$$
E\left[\exp \left(h \sum_{i=1}^{n} X_{i}\right)\right]=E\left[\prod_{i=1}^{n} \exp \left(h X_{i}\right)\right] \leq M \prod_{i=1}^{n} E\left[\exp \left(h X_{i}\right)\right] .
$$

Lemma 2.3 Let $\left\{X_{n}, n \geq 1\right\}$ be a sequence of ENOD random variables with mean zero and $0<B_{n}=\sum_{i=1}^{n} E X_{i}^{2}<\infty$. Then there exists a $M>0$ such that for all $x>0, y>0$,

$$
P\left(\left|S_{n}\right| \geq x\right) \leq \sum_{i=1}^{n} P\left(\left|X_{i}\right| \geq y\right)+2 M \exp \left(\frac{x}{y}-\frac{x}{y} \log \left(1+\frac{x y}{B_{n}}\right)\right),
$$

where $S_{n}=\sum_{i=1}^{n} X_{i}$.

Proof. The proof is similar to that of Theorem 2 in Fuk and Nagev(1971). Let $Y_{i}=X_{i} I\left(X_{i} \leq y\right)+y I\left(X_{i}>y\right)$ and $T_{n}=\sum_{i=1}^{n} Y_{i}$ and note that $Y_{i} \leq X_{i}$. It is easy to show that $E Y_{i} \leq 0$ and $E Y_{i}^{2} \leq E X_{i}^{2}$. By Lemma 2.1 (1) for $h>0\left\{e^{h Y_{i}}, 1 \leq i \leq n\right\}$ is a sequence of nonnegative ENOD random variables. Thus, by Lemma 2.2 there exists a constant $M>0$ such that

$$
E \exp \left(h T_{n}\right)=E \prod_{i=1}^{n} \exp \left(h Y_{i}\right) \leq M \prod_{i=1}^{n} E \exp \left(h Y_{i}\right) .
$$

Let $F_{i}(x)=P\left(X_{i}<x_{i}\right)$. Then, we have for $h>0$

$$
\begin{aligned}
& E \exp \left(h Y_{i}\right)=\int_{-\infty}^{y} \exp (h(x)) d F_{i}(x)+e^{h y} P\left(X_{i} \geq y\right) \\
= & 1+h E Y_{i}+\int_{-\infty}^{y}\left(e^{h x}-1-h x\right) d F_{i}(x)+\left(e^{h y}-1-h y\right) P\left(X_{i} \geq y\right) \\
\leq & 1+\int_{-\infty}^{y}\left(e^{h x}-1-h x\right) d F_{i}(x)+\left(e^{h y}-1-h y\right) P\left(X_{i} \geq y\right) .
\end{aligned}
$$


Since $f(x)=\left(e^{h x}-1-h x\right) / x^{2}$ is increasing for all $x, h>0$ and $1+u \leq e^{u}$ for all real number $u$ it follows from (2.4) that

$$
\text { (2.5) } \begin{aligned}
E \exp \left(h Y_{i}\right) & \leq 1+\frac{e^{h y}-1-h y}{y^{2}}\left(\int_{-\infty}^{y} x^{2} d F_{i}(x)+y^{2} P\left(X_{i} \geq y\right)\right) \\
& \leq 1+\frac{e^{h y}-1-h y}{y^{2}} E X_{i}^{2} \\
& \leq \exp \left(\frac{e^{h y}-1-h y}{y^{2}} E X_{i}^{2}\right) .
\end{aligned}
$$

Therefore by (2.3) and (2.5) we obtain, for all $x>0$ and all $h>0$

$$
\exp (-h x) E \exp \left(h T_{n}\right) \leq M \exp \left(-h x+B_{n} \frac{e^{h y}-1-h y}{y^{2}}\right) .
$$

Letting $h=\log \left(1+\frac{x y}{B_{n}}\right) / y$, we have

$$
\begin{aligned}
& \exp (-h x) E \exp \left(h T_{n}\right) \\
& \leq M \exp \left[\frac{x}{y}-\frac{x}{y} \log \left(1+\frac{x y}{B_{n}}\right)-\frac{B_{n}}{y^{2}} \log \left(1+\frac{x y}{B_{n}}\right)\right] \\
& \leq M \exp \left[\frac{x}{y}-\frac{x}{y} \log \left(1+\frac{x y}{B_{n}}\right)\right],
\end{aligned}
$$

which yields

$$
\text { 8) } \begin{aligned}
P\left(S_{n} \geq x\right) & \leq P\left(S_{n} \neq T_{n}\right)+P\left(T_{n} \geq x\right) \\
& \leq \sum_{i=1}^{n} P\left(X_{i} \geq y\right)+\exp (h x) E \exp \left(h T_{n}\right) \\
& \leq \sum_{i=1}^{n} P\left(X_{i} \geq y\right)+M \exp \left[\frac{x}{y}-\frac{x}{y} \log \left(1+\frac{x y}{B_{n}}\right)\right] .
\end{aligned}
$$

Similarly, we have

$$
P\left(-S_{n} \geq x\right) \leq \sum_{i=1}^{n} P\left(-X_{i} \geq y\right)+M \exp \left[\frac{x}{y}-\frac{x}{y} \log \left(1+\frac{x y}{B_{n}}\right)\right]
$$

since $\left\{-X_{n}, n \geq 1\right\}$ is a sequence of ENOD by Lemma 2.1 (1).

From (2.8) and (2.9) we obtain

$$
\begin{aligned}
P\left(\left|S_{n}\right| \geq x\right) & \leq P\left(S_{n} \geq x\right)+P\left(-S_{n} \geq x\right) \\
& \leq \sum_{i=1}^{n} P\left(\left|X_{i}\right| \geq y\right)+2 M \exp \left[\frac{x}{y}-\frac{x}{y} \log \left(1+\frac{x y}{B_{n}}\right)\right] .
\end{aligned}
$$




\section{Complete moment convergence}

Theorem 3.1 Let $\left\{X_{n i}, 1 \leq i \leq n, n \geq 1\right\}$ be an array of ENOD random variables with $E X_{n i}=0$ and $E X_{n i}^{2}<\infty$, for $1 \leq i \leq n, n \geq 1$ and $\left\{a_{n}, n \geq 1\right\}$ be a sequence of positive real numbers with $a_{n} \uparrow$. If

$$
\sum_{n=1}^{\infty} \sum_{i=1}^{n} \frac{E X_{n i}^{2}}{a_{n}^{2}}<\infty
$$

then we obtain

\section{Proof.}

$$
\sum_{n=1}^{\infty} a_{n}^{-1} E\left\{\left|\sum_{i=1}^{n} X_{n i}\right|-\epsilon a_{n}\right\}^{+}<\infty \text { for all } \epsilon>0 .
$$

$$
\begin{aligned}
\sum_{n=1}^{\infty} a_{n}^{-1} E\left\{\left|\sum_{i=1}^{n} X_{n i}\right|-\epsilon a_{n}\right\}^{+}= & \sum_{n=1}^{\infty} a_{n}^{-1} \int_{0}^{\infty}\left(P\left\{\left|\sum_{i=1}^{n} X_{n i}\right|-\epsilon a_{n}\right\}>u\right) d u \\
= & \sum_{n=1}^{\infty} a_{n}^{-1} \int_{0}^{a_{n}} P\left\{\left|\sum_{i=1}^{n} X_{n i}\right|>\epsilon a_{n}+u\right\} d u \\
& +\sum_{n=1}^{\infty} a_{n}^{-1} \int_{a_{n}}^{\infty} P\left\{\left|\sum_{i=1}^{n} X_{n i}\right|>\epsilon a_{n}+u\right\} d u \\
\leq & \sum_{n=1}^{\infty} P\left\{\left|\sum_{i=1}^{n} X_{n i}\right|>\epsilon a_{n}\right\} \\
& +\sum_{n=1}^{\infty} a_{n}^{-1} \int_{a_{n}}^{\infty} P\left\{\left|\sum_{i=1}^{n} X_{n i}\right|>u\right\} d u \\
= & I+I I .
\end{aligned}
$$

We need to prove that $I<\infty$ and $I I<\infty$.

For any $1 \leq i \leq n, n \geq 1$, let

$$
\begin{aligned}
Y_{n i} & =-a_{n} I\left(X_{n i}<-a_{n}\right)+X_{n i} I\left(\left|X_{n i}\right| \leq a_{n}\right)+a_{n} I\left(X_{n i}>a_{n}\right), \\
(3.3) Z_{n i} & =X_{n i}-Y_{n i} \\
& =\left(X_{n i}+a_{n}\right) I\left(X_{n i}<-a_{n}\right)+\left(X_{n i}-a_{n}\right) I\left(X_{n i}>a_{n}\right) .
\end{aligned}
$$

To prove $I<\infty$, it is enough to show that

$$
\begin{gathered}
\sum_{n=1}^{\infty} P\left(\frac{1}{a_{n}}\left|\sum_{i=1}^{n} Z_{n i}\right|>\epsilon\right)<\infty, \\
\sum_{n=1}^{\infty} P\left(\frac{1}{a_{n}}\left|\sum_{i=1}^{n}\left(Y_{n i}-E Y_{n i}\right)\right|>\epsilon\right)<\infty,
\end{gathered}
$$




$$
\frac{1}{a_{n}} \sum_{i=1}^{n} E Y_{n i} \rightarrow 0 \text { as } n \rightarrow \infty .
$$

Because the proof of (3.4) is a standard argument, we omit to prove (3.4). Now we prove (3.5).

By Lemma 2.1 (1) $\left\{Y_{n i}-E Y_{n i}, 1 \leq i \leq n, n \geq 1\right\}$ is an array of rowwise ENOD random variables with mean zero. Let $B_{n}^{\prime}=\sum_{i=1}^{n} E\left(Y_{n i}-\right.$ $\left.E Y_{n i}\right)^{2}<\infty$. Take $x=\epsilon a_{n}, y=\epsilon a_{n} / 2$. Then, by Lemma 2.3 , for all $\epsilon>0$

$$
\begin{aligned}
& \sum_{n=1}^{\infty} P\left(\frac{1}{a_{n}}\left|\sum_{i=1}^{n}\left(Y_{n i}-E Y_{n i}\right)\right|>\epsilon\right) \\
& \leq \sum_{n=1}^{\infty} \sum_{i=1}^{n} P\left(\left|Y_{n i}-E Y_{n i}\right|>\epsilon a_{n} / 2\right)+2 e^{2} M \sum_{n=1}^{\infty}\left(\frac{B_{n}^{\prime}}{B_{n}^{\prime}+\epsilon^{2} a_{n}^{2} / 2}\right)^{2} \\
& =I_{1}+I_{2} .
\end{aligned}
$$

Note that $\left|Y_{n i}\right| \leq\left|X_{n i}\right|$ and $E Y_{n i}^{2} \leq E X_{n i}^{2}$ for $i, 1 \leq i \leq n$.

By (3.1) and the Chebyshev's inequality

$$
\text { (3.8) } \begin{aligned}
I_{1} & \leq \sum_{n=1}^{\infty} \sum_{i=1}^{n} P\left(\left|Y_{n i}-E Y_{n i}\right|>\epsilon a_{n} / 2\right) \\
& \leq 4 \epsilon^{-2} \sum_{n=1}^{\infty} \sum_{i=1}^{n} \frac{E\left(Y_{n i}-E Y_{n i}\right)^{2}}{a_{n}^{2}} \\
& \leq 4 \epsilon^{-2} \sum_{n=1}^{\infty} \sum_{i=1}^{n} \frac{E X_{n i}^{2}}{a_{n}^{2}}<\infty .
\end{aligned}
$$

By (3.1) we obtain

$$
\text { (3.9) } \begin{aligned}
I_{2} & \leq 8 e^{2} \epsilon^{-4} M \sum_{n=1}^{\infty}\left(\sum_{i=1}^{n} a_{n}^{-2} E\left(Y_{n i}-E Y_{n i}\right)^{2}\right)^{2} \\
& \leq 8 e^{2} \epsilon^{-4} M \sum_{n=1}^{\infty}\left(\sum_{i=1}^{n} \frac{E Y_{n i}^{2}}{a_{n}^{2}}\right)^{2} \\
& \leq 8 e^{2} \epsilon^{-4} M \sum_{n=1}^{\infty}\left(\sum_{i=1}^{n} \frac{E X_{n i}^{2}}{a_{n}^{2}}\right)^{2} \\
& \leq 8 e^{2} \epsilon^{-4} M \sum_{n=1}^{\infty} \sum_{i=1}^{n} \frac{E X_{n i}^{2}}{a_{n}^{2}}<\infty .
\end{aligned}
$$


From (3.7)-(3.9) (3.5) follows.

To prove (3.6): Since $E X_{n i}=0$ for $1 \leq i \leq n, n \geq 1, E Y_{n i}=-E Z_{n i}$. If $X_{n i}>a_{n}, 0<Z_{n i}=X_{n i}-a_{n}<X_{n i}$ and if $X_{n i}<-a_{n}, X_{n i}<Z_{n i}=$ $X_{n i}+a_{n} \leq 0$. So $\left|Z_{n i}\right| \leq\left|X_{n i}\right| I\left(\left|X_{n i}\right|>a_{n}\right)$. Consequently

$$
\begin{aligned}
\frac{1}{a_{n}}\left|\sum_{i=1}^{n} E Y_{n i}\right| & =\frac{1}{a_{n}}\left|\sum_{i=1}^{n} E Z_{n i}\right| \\
& \leq \sum_{i=1}^{n} \frac{E\left|Z_{n i}\right|}{a_{n}} \\
& \leq \sum_{i=1}^{n} \frac{E a_{n}\left|X_{n i}\right| I\left(\left|X_{n i}\right|>a_{n}\right)}{a_{n}^{2}} \\
& \leq \sum_{i=1}^{n} \frac{E X_{n i}^{2}}{a_{n}^{2}} \rightarrow 0 \text { as } n \rightarrow \infty \text { by }(3.1) .
\end{aligned}
$$

Hence the proof of $I<\infty$ is complete.

Next we prove that $I I<\infty$.

$$
\begin{aligned}
I I \leq & \sum_{n=1}^{\infty} a_{n}^{-1} \sum_{i=1}^{n} \int_{a_{n}}^{\infty} P\left\{\left|X_{n i}\right|>u\right\} d u \\
& +\sum_{n=1}^{\infty} a_{n}^{-1} \sum_{i=1}^{n} \int_{a_{n}}^{\infty} P\left\{\left|\sum_{i=1}^{n} I\left(\left|X_{n i}\right| \leq u\right)\right|>u\right\} d u \\
= & I I_{1}+I I_{2} .
\end{aligned}
$$

Clearly, for $u \geq a_{n}$ we have

$$
\begin{aligned}
I I_{1} & =\sum_{n=1}^{\infty} \sum_{i=1}^{n} a_{n}^{-1} \int_{a_{n}}^{\infty} P\left\{\left|X_{n i}\right|>u\right\} d u \\
& \leq \sum_{n=1}^{\infty} \sum_{i=1}^{n} a_{n}^{-1} \int_{0}^{\infty} P\left\{\left|X_{n i}\right| I\left(\left|X_{n i}\right|>a_{n}\right)>u\right\} d u \\
& =\sum_{n=1}^{\infty} \sum_{i=1}^{n} \frac{E\left|X_{n i}\right| I\left(\left|X_{n i}\right|>a_{n}\right)}{a_{n}} \\
& \leq \sum_{n=1}^{\infty} \sum_{i=1}^{n} \frac{E X_{n i}^{2}}{a_{n}^{2}}<\infty \text { by }(3.1) .
\end{aligned}
$$


It remains to prove $I I_{2}<\infty$. It follows from $E X_{n i}=0$ and (3.1) that

$$
\begin{aligned}
\max _{u \geq a_{n}}\left|u^{-1} \sum_{i=1}^{n} E X_{n i} I\left(\left|X_{n i}\right| \leq u\right)\right| & =\max _{u \geq a_{n}}\left|u^{-1} \sum_{i=1}^{n} E X_{n i} I\left(\left|X_{n i}\right|>u\right)\right| \\
& \leq \sum_{i=1}^{n} \frac{E\left|X_{n i}\right| I\left(\left|X_{n i}\right|>a_{n}\right)}{a_{n}} \\
& \leq \sum_{i=1}^{n} \frac{E X_{n i}^{2}}{a_{n}^{2}} \rightarrow 0 \text { by }(3.1) .
\end{aligned}
$$

Therefore, while $n$ is sufficiently large, for $u \geq a_{n}$,

$$
\left|\sum_{i=1}^{n} E X_{n i} I\left(\left|X_{n i}\right| \leq u\right)\right| \leq \frac{u}{2}
$$

which yields

$$
\begin{aligned}
& P\left\{\left|\sum_{i=1}^{n} X_{n i} I\left(\left|X_{n i}\right| \leq u\right)\right|>u\right\} \\
\leq & P\left\{\left|\sum_{i=1}^{n}\left(X_{n i} I\left(\left|X_{n i}\right| \leq u\right)-E X_{n i} I\left(\left|X_{n i}\right| \leq u\right)\right)\right|>\frac{u}{2}\right\} .
\end{aligned}
$$

Let $B_{n}^{\prime \prime}=\sum_{i=1}^{n} E\left(X_{n i}-E X_{n i}\right)^{2} I\left(\left|X_{n i}\right| \leq u\right), x=\frac{u}{2}, y=\frac{u}{4}$. By (3.10) and Lemma 2.3 we get

$$
\begin{aligned}
I I_{2} \leq & \sum_{n=1}^{\infty} a_{n}^{-1} \int_{a_{n}}^{\infty} P\left\{\left|\sum_{i=1}^{n} X_{n i} I\left(\left|X_{n i}\right| \leq u\right)-E X_{n i} I\left(\left|X_{n i}\right| \leq u\right)\right|>\frac{u}{2}\right\} d u \\
\leq & \sum_{n=1}^{\infty} a_{n}^{-1} \int_{a_{n}}^{\infty} \sum_{i=1}^{n} P\left\{\left|X_{n i}\right| I\left(\left|X_{n i}\right| \leq u\right)-E X_{n i} I\left(\left|X_{n i}\right| \leq u\right) \mid>\frac{u}{4}\right\} d u \\
& +2 e^{2} M \sum_{n=1}^{\infty} a_{n}^{-1} \int_{a_{n}}^{\infty}\left(\frac{B_{n}^{\prime \prime}}{B_{n}^{\prime \prime}+\frac{u^{2}}{8}}\right)^{2} d u \\
= & I I_{21}+I I_{22} .
\end{aligned}
$$

By (3.1) and $E X_{n i}=0$ we also have

$$
\begin{aligned}
\max _{u \geq a_{n}} u^{-1}\left|E X_{n i} I\left(\left|X_{n i}\right| \leq u\right)\right| & =\max _{u \geq a_{n}} u^{-1}\left|E X_{n i} I\left(\left|X_{n i}\right|>u\right)\right| \\
& \leq a_{n}^{-1} E\left|X_{n i}\right| I\left(\left|X_{n i}\right|>a_{n}\right) \mid \\
& \leq \sum_{i=1}^{n} \frac{E X_{n i}^{2}}{a_{n}^{2}} \rightarrow 0
\end{aligned}
$$


Hence,

$$
\begin{aligned}
I I_{21} \leq & \sum_{n=1}^{\infty} \sum_{i=1}^{n} a_{n}^{-1} \int_{a_{n}}^{\infty} P\left\{\left|X_{n i}\right| I\left(\left|X_{n i}\right| \leq u\right)>\frac{u}{8}\right\} d u \\
= & \sum_{n=1}^{\infty} \sum_{i=1}^{n} a_{n}^{-1} \int_{a_{n}}^{\infty} P\left\{\left|X_{n i}\right| I\left(\left|X_{n i}\right| \leq a_{n}\right)>\frac{u}{8}\right\} d u \\
& +\sum_{n=1}^{\infty} \sum_{i=1}^{n} a_{n}^{-1} \int_{a_{n}}^{\infty} P\left\{\left|X_{n i}\right| I\left(a_{n}<\left|X_{n i}\right| \leq u\right)>\frac{u}{8}\right\} d u \\
= & I I_{211}+I I_{212} .
\end{aligned}
$$

By the Chebyshev's inequality and (3.1) we have

$$
\begin{aligned}
I_{211} & \leq 64 \sum_{n=1}^{\infty} \sum_{i=1}^{n} a_{n}^{-1} E\left|X_{n i}\right|^{2} I\left(\left|X_{n i}\right| \leq a_{n}\right) \int_{a_{n}}^{\infty} u^{-2} d u \\
& \leq 64 \sum_{n=1}^{\infty} \sum_{i=1}^{n} \frac{E\left|X_{n i}\right|^{2} I\left(\left|X_{n i}\right| \leq a_{n}\right)}{a_{n}^{2}}<\infty .
\end{aligned}
$$

By the similar method to proof of $I I_{1}$

$$
\begin{aligned}
I I_{212} & =\sum_{n=1}^{\infty} \sum_{i=1}^{n} a_{n}^{-1} \int_{a_{n}}^{\infty} P\left\{\left|X_{n i}\right| I\left(a_{n}<\left|X_{n i}\right| \leq u\right) \mid>\frac{u}{8}\right\} d u \\
& \leq \sum_{n=1}^{\infty} \sum_{i=1}^{n} a_{n}^{-1} \int_{0}^{\infty} P\left\{\left|X_{n i}\right| I\left(\left|X_{n i}\right|>a_{n}\right) \mid>\frac{u}{8}\right\} d u \\
& =8 \sum_{n=1}^{\infty} \sum_{i=1}^{n} \frac{E\left|X_{n i}\right| I\left(\left|X_{n i}\right|>a_{n}\right)}{a_{n}} \\
& \leq 8 \sum_{n=1}^{\infty} \sum_{i=1}^{n} \frac{E X_{n i}^{2}}{a_{n}^{2}}<\infty .
\end{aligned}
$$


Finally, we have to prove $I I_{22}<\infty$. By the fact that for $x \geq 0, y \geq 0$ $(x+y)^{2} \leq 2\left(x^{2}+y^{2}\right)$

$$
\begin{aligned}
I I_{22}= & 2 e^{2} M \sum_{n=1}^{\infty} a_{n}^{-1} \int_{a_{n}}^{\infty}\left(\frac{B_{n}^{\prime \prime}}{B_{n}^{\prime \prime}+\frac{u^{2}}{8}}\right)^{2} d u \\
\leq & 128 e^{2} M \sum_{n=1}^{\infty} a_{n}^{-1} \int_{a_{n}}^{\infty}\left(\frac{B_{n}^{\prime \prime}}{u^{2}}\right)^{2} d u \\
\leq & 128 e^{2} M \sum_{n=1}^{\infty} a_{n}^{-1} \int_{a_{n}}^{\infty}\left(\sum_{i=1}^{n} \frac{E X_{n i}^{2} I\left(\left|X_{n i}\right| \leq a_{n}\right)}{u^{2}}\right. \\
& \left.+\sum_{i=1}^{n} \frac{E X_{n i}^{2} I\left(a_{n}<\left|X_{n i}\right| \leq u\right)}{u^{2}}\right)^{2} d u \\
\leq & 256 e^{2} M \sum_{n=1}^{\infty} a_{n}^{-1} \int_{a_{n}}^{\infty}\left(\sum_{i=1}^{n} \frac{E X_{n i}^{2} I\left(\left|X_{n i}\right| \leq a_{n}\right)}{u^{2}}\right)^{2} d u \\
& +256 e^{2} M \sum_{n=1}^{\infty} a_{n}^{-1} \int_{a_{n}}^{\infty}\left(\frac{E X_{n i}^{2} I\left(a_{n}<\left|X_{n i}\right| \leq u\right)}{u^{2}}\right)^{2} d u \\
= & I I_{221}+I I_{222} .
\end{aligned}
$$

Let $C=256 e^{2} M$.

$$
\begin{aligned}
I I_{221} & =C \sum_{n=1}^{\infty} a_{n}^{-1}\left(\sum_{i=1}^{n} E X_{n i}^{2} I\left(\left|X_{n i}\right| \leq a_{n}\right)\right)^{2} \int_{a_{n}}^{\infty} u^{-4} d u \\
& \leq \frac{C}{3} \sum_{n=1}^{\infty} a_{n}^{-4}\left(\sum_{i=1}^{n} E X_{n i}^{2} I\left(\left|X_{n i}\right| \leq a_{n}\right)\right)^{2} \\
& \leq \frac{C}{3} \sum_{n=1}^{\infty}\left(\sum_{i=1}^{n} \frac{E\left|X_{n i}\right|^{2} I\left(\left|X_{n i}\right| \leq a_{n}\right)}{a_{n}^{2}}\right)^{2} \\
& \leq \frac{C}{3} \sum_{n=1}^{\infty}\left(\sum_{i=1}^{n} \frac{E\left(X_{n i}\right)^{2} I\left(\left|X_{n i}\right| \leq a_{n}\right)}{a_{n}^{2}}\right)^{2}<\infty .
\end{aligned}
$$




$$
\begin{aligned}
I I_{222} & \leq C \sum_{n=1}^{\infty} a_{n}^{-1} \int_{a_{n}}^{\infty}\left(\sum_{i=1}^{n} \frac{E\left|X_{n i}\right| I\left(a_{n}<\left|X_{n i}\right| \leq u\right)}{u}\right)^{2} d u \\
& \leq C \sum_{n=1}^{\infty} a_{n}^{-1} \int_{a_{n}}^{\infty}\left(\sum_{i=1}^{n} \frac{E\left|X_{n i}\right| I\left(\left|X_{n i}\right|>a_{n}\right)}{u}\right)^{2} d u \\
& \leq C \sum_{n=1}^{\infty} a_{n}^{-1}\left(\sum_{i=1}^{n} E\left|X_{n i}\right| I\left(\left|X_{n i}\right|>a_{n}\right)\right)^{2} \int_{a_{n}}^{\infty} u^{-2} d u \\
& \leq C \sum_{n=1}^{\infty}\left(\sum_{i=1}^{n} \frac{E\left|X_{n i}\right| I\left(\left|X_{n i}\right|>a_{n}\right)}{a_{n}}\right)^{2} \\
& \leq C \sum_{n=1}^{\infty}\left(\sum_{i=1}^{n} \frac{E X_{n i}^{2} I\left(\left|X_{n i}\right|>a_{n}\right)}{a_{n}^{2}}\right)^{2} \\
& \leq C \sum_{n=1}^{\infty} \sum_{i=1}^{n}\left(\frac{E X_{n i}^{2}}{a_{n}^{2}}\right)^{2} \\
& \leq C\left(\sum_{n=1}^{\infty} \sum_{i=1}^{n} \frac{E X_{n i}^{2}}{a_{n}^{2}}\right)^{2}<\infty .
\end{aligned}
$$

Remark In the proof of Theorem 3.1 from the fact that $I<\infty$ we obtain the complete convergence $\sum_{i=1}^{n} \frac{X_{n i}}{a_{i}} \rightarrow 0$.

Corollary 3.2 Let $\left\{X_{n i}, 1 \leq i \leq n, n \geq 1\right\}$ be an array of POD random variables with $E X_{n i}=0$ and $E X_{n i}^{2}<\infty$ and $\left\{a_{n}, n \geq 1\right\}$ be a sequence positive real numbers with $a_{n} \uparrow \infty$. If there exists a constant $M>$ 1 satisfying (1.4) and condition (3.1) then $\sum_{n=1}^{\infty} a_{n}^{-1} E\left\{\left|\sum_{i=1}^{n} X_{n i}\right|-\right.$ $\left.\epsilon a_{n}\right\}_{+}<\infty$.

\section{References}

[1] Ebrahimi, N. and Ghosh, M.(1981) Multivariate negative dependence, Commun, Statist Theor. Meth. 10 307-337

[2] Chow, Y.S.(1988) On the rate of moment complete convergence of sample sums and extremes, Bull. Inst. Math. Acad. Sinica 16 177-201

[3] Fuk, D.K. and Nagaev, S.V.(1971) Probability inequalities for sums of independent random variables, Theor. Probab. Apple. 16 643-660

[4] Hsu, P.L. and Robbins, H.(1947) Complete convergence and the law of large numbers, Proc. Nat. Acad. Sci. U.S.A. 33 25-31 
[5] Joag-Dev, K. and Proschan, F.(1983) Negative association of random variables with applications, Ann. Statist.

$11286-295$

[6] Liu, L.(2009) Precise large deviations for dependent random variables with heavy tails, Statist. Probab. Letts. 99 1290-1298

[7] Wang, D.C. and Zhao, W.(2006) Moment complete convergence for sums of a sequence NA random variables, Appl. Math. J. Chinese Univ. 21 445-450(in Chinese)

[8] Zhu, M.H.(2007) Strong laws of large numbers for arrays of rowwise $\rho^{*}$-mixing random variables, Discrete Dynamics in Nature Soc. Vol. 2007 Article ID 74296, 6 pages 2007

\section{Dae-Hee Ryu}

Department of Computer Science, ChungWoon University,

Hongseong 351-701, Korea.

E-mail:rdh@chungwoon.ac.kr 